\pm 237 and $570 \pm 217 \mathrm{mU} / \mathrm{dL}(\mathrm{p}=0.674) ;$ LS-BMD $0.85 \pm 0.16$ and 0.74 $\pm 0.13 \mathrm{~g} / \mathrm{cm}^{2}(\mathrm{p}<0.001)$ and TH-BMD $0.62 \pm 0.09$ and $0.60 \pm 0.08 \mathrm{~g} / \mathrm{cm}^{2}$ $(p=0.110)$. The rate of decreased P1NP from baseline to 6,12 and 24 months were each $-43.7 \%$ vs $-61.3 \%(p=0.015)$ at 6 month, $-40.2 \%$ vs $55.2 \%(p<0.001)$ at 12 month and $-43.5 \%$ vs $-67.9 \%(p<0.001)$ at 24 month and TRAC- $5 \mathrm{~b}$ were $-44.6 \%$ vs $-51.5 \% \quad(\mathrm{p}=0.225)$ at 6 month, $39.5 \%$ vs $-53.0 \% \quad(p=0.007)$ at 12 month and $-41.3 \%$ vs $-54.2 \%$ $(p=0.663)$ at 24 month in the RA-OP vs P-OP group. The rate of increased LS-BMD from baseline to 6,12 and 24 months were each $4.3 \%$ vs $4.5 \%(p=0.743)$ at 6 month, $6.3 \%$ vs $6.9 \% \quad(p=0.575)$ at 12 month and $9.3 \%$ vs $11.1 \%(\mathrm{p}=0.300)$ at 24 month and TH-BMD were $2.5 \%$ vs $2.6 \%(p=0.583)$ at 6 month, $3.3 \%$ vs $3.2 \% \quad(p=0.683)$ at 12 month and $5.7 \%$ vs $4.2 \%(\mathrm{p}=0.403)$ at 24 month RA-OP vs P-OP group. Conclusion: DMB was effective for both RA-OP and P-OP, and the clinical efficacy was almost similar between the two groups.

Disclosure of Interests: None declared

DOI: 10.1136/annrheumdis-2019-eular.6952

\section{FRI0494 CORRELATION OF METACARPAL BONE MASS MEASURED EITHER BY DUAL X-RAY DENSITOMETRY OR DIGITAL X-RAY RADIOGRAMMETRY WITH SEVERITY OUTCOMES IN PATIENTS WITH EARLY ARTHRITIS}

Irene Llorente ${ }^{1}$, Saturnino Gonzalez ${ }^{2}$, Eugenio Escolano ${ }^{2}$, Ana Maria Ortiz ${ }^{1}$, Alberto García-Vadillo ${ }^{1}$,Isidoro González-Álvaro ${ }^{1}$, Santos Castañeda ${ }^{1}$. ${ }^{1}$ University Hospital La Princesa, Rheumatology Department, Madrid, Spain; ${ }^{2}$ University Hospital La Princesa, Radiology department, Madrid, Spain

Background: Rheumatoid arthritis (RA) is a systemic autoimmune disorder that predominantly affects small joints of the hands and feet. X-ray digital radiogrammetry (DXR) is a validated technique for the evaluation of bone mineral density (BMD) in the diaphysis of metacarpals (MC) that has demonstrated that low bone mass at this location correlates with worse radiographic progression (1). However, this technique is not available in our environment. Dual X-ray densitometry (DXA) is a simple, accessible and widely validated technique for the study of osteoporosis. Our group has previously validated the reproducibility of MCP measurements by DXA (2).

Objectives: To study the relationship between baseline MC BMD measured by DXA or DXR and disease severity in patients with early arthritis at 2 years follow-up.

Methods: 202 patients belonging to PEARL (Princess Early Arthritis register longitudinal) study were included. Demographic, laboratory, radiographic and therapeutic data were recorded by protocol. Most patients (87\%) were women, 59\% fulfilled 2010 ACR criteria and $41 \%$ were classified as undifferentiated arthritis. More than $60 \%$ were seropositive (60\% RF; $58 \%$ anti-(CP). Median disease duration at first visit was 4.6 months, median DAS28 4.2 and HAQ 0.875. The BMD of 2 th to $4^{\text {th }}$ MC was measured by DXR through the standardized software by Sectra (Linköping, Sweden) applied on radiographs of hands submitted in digital format (GEC) DX Definium 8000). Baseline non-dominant hand DXA was performed using a densitometer Hologice QDR4500. To analyze $2^{\text {th }}$ to $4^{\text {th }}$ MC BMD, we created 3 regions of interest $(\mathrm{ROI})$ similar to that generated in the DXR measurements; briefly, the ROls cover the mid-third of each MC diaphysis, avoiding overlapping of ROls. The statistical study was performed with Stata 12 for Windows, including linear correlation between MC-BMD values measured by DXR and DXA (Pearson's test), and different multivariate analyses in which MC-BMD measured either by DXR or DXA were included as dependent variable and sex, age and body mass index (BMI) as independent confounding variables. Then in these models different subrogate variables of disease severity such as disease activity (DAS28), disability (HAQ) or cumulative DMARD treatment after 2 years of follow-up were included in order to determine whether there was an association with MC-BMD.

Results: The MC-BMD measured by DXA and DXR at baseline showed a good correlation $(r=0.87, p<0.001)$. As expected, being female ( $B$ coefficient $-0.0381, \mathrm{p}=0.0001$ ) and age $>65$ years $(B$ coefficient -0.076 , $\mathrm{p}=0.0001$ ) were associated with lower $\mathrm{BMD}$, whereas high body mass index ( $B$ coefficient $0.002 \times \mathrm{kg} / \mathrm{m}^{2}, p=0.004$ ) was associated with higher BMD. Adjusting by this confounding variables, the multivariate analyses did not show any significant association between MC-BMD and DAS28, HAQ or treatment intensity, neither with the DXA or DXR measurements. Conclusion: Despite of the good correlation between DXR and DXA, our data obtained either with DXA or DXR do not confirm the association of low MC-BMD measured with DXR and more aggressive disease in patients with early arthritis.
REFERENCES:

[1] Forslind K, Boonen A, Albertsson $\mathrm{K}$, et al. Scand J Rheumatol 2009;38:431-438.

[2] Castañeda S, González-Álvaro I, Rodríguez-Salvanés F, et al. J Clin Densitom 2007;10:298-305.

Acknowledgement: We would like to thank Teresa Velasco, nurse of the Rheumatology Division, for her invaluable help in the Early Arthritis Clinic Disclosure of Interests: Irene Llorente : None declared, Saturnino Gonzalez: None declared, Eugenio Escolano: None declared, Ana Maria Ortiz: None declared, Alberto García-Vadillo: None declared, Isidoro GonzálezÁlvaro: None declared, Santos Castañeda Consultant for: Amgen, BMS, Pfizer, Lilly, MSD, Roche, Sanofi, UCB

DOI: 10.1136/annrheumdis-2019-eular.6271

\section{FRI0495 FRAGILITY FRACTURE IN MALE; EXPERIENCE OF A FRACTURE LIAISON SERVICE}

Antonio Naranjo ${ }^{1}$, Soledad Ojeda ${ }^{2}$, Aida Saavedra ${ }^{2}$, Cristina Sepúlveda ${ }^{2}$, Francisco Rubiño ${ }^{2}$, Amparo Molina ${ }^{2}$, Olga Suárez $z^{2}$, Casimira Domínguez ${ }^{2}$, José A. Lorenzo ${ }^{2}$, Nieves Martín ${ }^{2}$, Carlos Rodriguez-Lozano ${ }^{2} .{ }^{1}$ Las Palmas de Gran Canaria, Rheumatology, Las Palmas de Gran Canaria, Spain; ${ }^{2}$ Hospital universitario Dr Negrin, Rheumatology, Las Palmas de Gran Canaria, Spain

Background: Secondary prevention of osteoporotic fracture is more efficient with the Fracture Liaison Service (FLS) model.

Objectives: To describe the characteristics of male patients attended in a FLS

Methods: Prospective observational study. The program consists of: 1) recruitment from the emergency records, hip fracture admitted or referral to outpatient rheumatology; 3) baseline visit: questionnaire including demographCl variables, FRAX and previous treatments; 4) DXA; 5) patient education; 6) report of results to the patient and the PC doctor, with specifCl management recommendations; complex patients are referred to rheumatology; and 7) follow-up through telephone survey plus prescription check in electronCl records. The characteristCls of male patients are described and compared with women.

Results: 2,135 patients have been included, 382 male (18\%). The recruitment was in 300 cases (78\%) from the emergency list, in 55 cases (14\%) admission for hip fracture and in 25 cases (6\%) from outpatient clinCl. The characteristCls of the patients are shown in the table.

The average age was similar in men and women. Hip fracture was more frequent in men (34\% vs. $25 \%$, OR $1.63 \mathrm{Cl} 95 \% 1.50-1.75)$ as opposed to forearm fracture ( $18 \%$ vs. $33 \%$, OR $0.46 \mathrm{Cl} 95 \% 0.32-0.60)$. Male had previous DXA ( $6 \%$ vs $25 \%$, OR $0.22 \mathrm{Cl} 95 \% \quad 0.00-0.43)$ and previous treatment with bisphosphonate (4\% vs $19 \%$, OR $0.17 \mathrm{Cl} 95 \%$ 0, $0-0.44$ ) less frequently.

Of the variables included in the FRAX, in men was less frequent the previous fracture $(13 \%$ vs $24 \%$, OR $0.48 \mathrm{Cl} 95 \% 0.32-0.64)$, the hip fracture of the parents ( $9 \%$ vs. $13 \%$; OR $0.66 \mathrm{Cl} 95 \% 0.47-0.85)$ and the use of corticoids (6\% vs $10 \%$, OR $0.61 \mathrm{Cl} 95 \%$ 0.39-0.84). However active smoking was more frequent in men $(21 \%$ vs $14 \%$, OR $1.67 \mathrm{Cl}$ $95 \% 1.53-1.81)$ as well as consumption $>2$ daily units of alcohol $(18 \%$ vs $5 \%$; OR $3.96 \mathrm{Cl} 95 \% 3.78-4.13$ ).

The percentage of osteoporosis was lower in men compared to women (30\% vs $46 \%$, OR $0.49 \mathrm{Cl} 95 \% 0.30-0.68$ ) as well as the percentage of patients with $>1$ fall in the last year $(29 \%$ vs $45 \%$, OR $0.51 \mathrm{Cl} 95 \%$ 0.30-0.71).

In addition, after the visit to the FLS, a bisphosphonate was indicated less frequently to men than to women $(68 \%$ vs. $77 \%$, OR $0.64 \mathrm{Cl} 95 \%$ $0.51-0.76)$ and were referred less frequently to PC (64\% vs. $76 \%$; OR $0.5595 \% \mathrm{Cl}$ 0.41-0.70). Finally, persistence at 12 months was lower in men than in women $(53 \%$ vs. $68 \%$, OR $0.53 \mathrm{Cl} 95 \%$ 0.30-0.76).

Conclusion: Compared with women, men seen in an FLS present hip fracture more frequently and a forearm fracture less frequently. Men also have a lower frequency of personal and family history of fracture and a greater frequency of smoking and alcohol intake. The percentage of patients who are candidates for treatment and persistence is lower than in women. These data should be taken into account in the identification and treatment of fragility fracture in men.

Acknowledgement: Fabiola Santana and Carmen Alonso

Disclosure of Interests: Antonio Naranjo Grant/research support from: Amgen, Consultant for: UCB, Speakers bureau: Amgen, UCB, Soledad Ojeda Grant/research support from: AMGEN, Speakers bureau: AMGEN, Aida Saavedra: None declared, Cristina Sepúlveda: None declared, Francisco Rubiño: None declared, Amparo Molina Speakers bureau: AMGEN, Olga Suárez: None declared, Casimira Domínguez: None declared, José 
A. Lorenzo: None declared, Nieves Martín: None declared, Carlos Rodriguez-Lozano: None declared

DOI: 10.1136/annrheumdis-2019-eular.928

Table

\begin{tabular}{lc}
\hline Age, mean (SD) & $73.4(10)$ \\
\hline BMI, mean & 27.4 \\
Fracture type, N (\%) & \\
Forearm & $71(18)$ \\
Hip & $132(34)$ \\
Humerus & $77(20)$ \\
Vertebra & $52(13)$ \\
Others & $49(13)$ \\
FRAX items, N (\%) & \\
Previous fracture & $51(13)$ \\
Hip fracture of parents & $36(9)$ \\
Smoking & $81(21)$ \\
Corticoids & $24(6)$ \\
Rheumatoid arthritis & $4(1)$ \\
Secondary osteoporosis & $17(4)$ \\
Alcohol & $68(18)$ \\
Densitometry (\%) & \\
Normal & 30 \\
Osteopenia & 43 \\
Osteoporosis & 26 \\
FRAX, mean (SD) & \\
Major fracture & \\
Hip fracture & $8,4(5)$ \\
Treatment prescribed, (\%) & $4,3(4)$ \\
Bishoshonate or equivalent & \\
Refferal to primary care & 68 \\
Persistence at 12 months & 64 \\
\hline
\end{tabular}

\section{FRI0496 OSTEOPOROSIS IN PRIMARY CARE - ARE WE MISSING A TRICK?}

Sultana Parvin ${ }^{1}$, Manraj Barhey ${ }^{2}$, Talib Abubacker ${ }^{3}$, Muhammad Khurram Nisar ${ }^{1}$. ${ }^{1}$ Luton and Dunstable University Hospital, Rheumatology, Luton, United Kingdom; ${ }^{2}$ Woodland Avenue Practice, Primary car, Luton, United Kingdom; ${ }^{3}$ Bell House Medical centre, Primary care, Luton, United Kingdom

Background: Globally various incentive schemes have been employed in primary care to improve early diagnosis and management of several rheumatic conditions. In the UK, the Primary Care Quality and Outcomes Framework (QOF) rewards general practices for the provision of 'quality care' and helps to fund further improvements in the delivery of clinical care. Currently, there is one quality indicator in place for secondary prevention of osteoporosis. In order to help establish an integrated care pathway encompassing the whole patient journey between primary and secondary care, we undertook a detailed survey of two GP practices. Objectives: The aims of the exercise were to identify the utility of quality indicator and any gaps in the model of care for the high-risk osteoporosis patients.

Methods: An independent service evaluation tool was employed to interrogate the IT system used in the GP surgeries. All patients over the age of 65 were extracted from the database and FRAX analysis was undertaken. Those with medium to high FRAX score (i.e. ten-year risk of $>20 \%$ for major osteoporotic fracture and/or $>5 \%$ for hip fracture) were captured to explore whether they were offered further evaluation and bone-sparing therapy as necessary.

Results: Of 18,248 patients registered in the multi-cultural urban practices, 6796 were $>65$ years old. 793 had pre-defined moderate-high FRAX score. $300(37 \%)$ had a confirmed diagnosis of osteoporosis. Median age was 78 (range 65-103 years). 249 (83\%) were women. 88.5\% were White and remaining of other ethnicities.

$20 / 300(6.6 \%)$ had been coded to have ever sustained a fragility fracture. $178(59.3 \%)$ were prescribed bone-sparing therapy with five people taking it for over five years. $91 \%$ were prescribed oral therapy $(78 \%$ alendronic acid, $10 \%$ risedronic acid and $3 \%$ others) and remaining had parenteral therapies. Of the 27 patients not receiving any treatment, $11(30 \%)$ were incorrectly coded. The remainder's reasons for lack of treatment include intolerance, poor adherence and comorbidities.

Conclusion: This study highlights the inadequacy of quality indicators in the overall management of osteoporosis burden in primary care. It relies heavily on active identification process for high-risk individuals and correct coding of fragility fracture. However the vast majority of patients with moderate-high risk, based on case finding strategy advised by international bodies e.g. FRAX, remain hidden. Less than $10 \%$ of patients with confirmed osteoporosis fulfil the quality outcome in this survey. The QOF hence fails to reflect the nature of disease burden in the primary care thereby risking the management strategies skewed towards too small a cohort and missing the big picture. It is clear that quality indicators for osteoporosis need to be aligned to risk stratification model. This will allow better identification of at-risk individuals and improved care pathway for patients requiring bone active therapies.

Disclosure of Interests: Sultana Parvin: None declared, Manraj Barhey: None declared, Talib Abubacker: None declared, Muhammad Khurram Nisar Grant/research support from: Muhammad Nisar undertakes clinical trials and received support (including attendance at conferences, speaker fees and honoraria) from Roche, Chugai, MSD, Abbvie, Pfizer, BMS, Novartis, Celgene, Mallinckrodt, UCB and Lilly, Consultant for: Muhammad Nisar undertakes clinical trials and received support (including attendance at conferences, speaker fees and honoraria) from Roche, Chugai, MSD, Abbvie, Pfizer, BMS, Novartis, Celgene, Mallinckrodt, UCB and Lilly, Speakers bureau: Muhammad Nisar undertakes clinical trials and received support (including attendance at conferences, speaker fees and honoraria) from Roche, Chugai, MSD, Abbvie, Pfizer, BMS, Novartis, Celgene, Mallinckrodt, UCB and Lilly

DOI: 10.1136/annrheumdis-2019-eular.206

\section{FRI0497 TRABECULAR BONE SCORE AND MALNUTRITION IN A COHORT OF SYSTEMIC SCLEROSIS PATIENTS}

Sabrina Paolino ${ }^{1}$, Massimo Patanè ${ }^{1}$, Veronica Tomatis ${ }^{1}$, Andrea Casabella ${ }^{1}$ Carmen Pizzorni ${ }^{1}$, Carlotta Schenone ${ }^{1}$, Luca Carmisciano ${ }^{2}$, Alessio Signori ${ }^{2}$, Maurizio Cutolo'. 'IRCCS San Martino Polyclinic Hospital, Genoa, Italy, Research Laboratory and Academic Division of Clinical Rheumatology, Department of Internal Medicine, University of Genova, genoa, Italy; ${ }^{2}$ IRCCS San Martino Polyclinic Hospital, Genoa, Italy, 2Biostatistics Unit, Department of Health Sciences, University of Genoa, Genoa, Italy, genoa, Italy

Background: Systemic sclerosis (SSc) is a connective tissue disease characterized by initial microvascular damage, immune system activation and progressive fibrosis of the skin and internal organs.Gastrointestinal (Gl) involvement induce malnutrition due to gastroesophageal symptoms, Gl dismotility and malabsorption that are related to fibrosis of bowel wall and bacterial overgrowth(1).Therefore the disease is associated with secondary osteoporosis with a few studies evaluating the bone microarchitecture(2).

Objectives: To evaluate a relationship between malnutrition and bone microarchitecture detected by trabecular bone score (TBS) in SSc patients.

Methods: 38 patients (6 male and 32 female) fulfilling ACR 2013 criteria for SSc underwent DXA to detect quantitative lumbar spine bone mineral density and TBS. DXA also assess body composition with a software that provides the physician quantitative parameters, including free fat mass index (FFMI), that identifies the patient with malnutrition(values $<15$ $\mathrm{kg} / \mathrm{m} 2$ in women and $17 \mathrm{~kg} / \mathrm{m} 2$ in men), according to the ESPEN criteria (3).Body mass index was calculated for all SSc patients and every patient completed a diary reporting Gl symptoms possibly related to intestinal disbiosis.Fasting blood samples were obtained in order to analyse some biochemical parameters of malnutrition (total proteins $(\mathrm{g} / \mathrm{L})$, albumin $(\mathrm{g} /$ $\mathrm{L})$, serum total cholesterol $(\mathrm{mg} / \mathrm{dl})$ and blood lymphocyte count $(\mathrm{N} / \mathrm{mm} 3)$. Continue variables were summarized as mean and standard deviation(SD) or median and inter quartile rang (IQR), discrete variables were summarized with count and percentage. Correlation was tested with Pearson or Spearman method. T-test was used to compare TBS between dichotomic groups Uni and multivariate linear regression models were used as well the Multiple R-squared variation was applied. The multivariate linear regression was performed with a stepwise approach to select the best model using highest AIC criteria.

Results: The mean age of patients was $64.2 \pm 11.3$ years with mean dis ease duration $19.2 \pm 7.6$ years. $36.8 \%$ of patients was found malnourished. The univariate analysis showed that only higher age of patients correlated to lower TBS $(p<0.001)$. The $R$-squared of multivariate linear regression showed that about $45 \%$ of the TBS variations(TBSv) can be explained by the variation of the following variables(age, disease duration, lymphocyte count).Age explains about $25 \%$ of the TBSv.Older patients had lower TBS, with approximately 0.05 points of TBS loss every $\operatorname{decade}(\mathrm{p}=$ 0.001). The presence of symptom possibly related to intestinal disbiosis, added to the model, might explains about $12 \%$ more of TBSv. Patients with symptom related to bacterial overgrowth had lower TBS respect to patients without(-0.08), regardless of other variables $(p=0.002)$. Disease duration, added to the model, further explains about $4 \%$ more of TBSv and suggest a trend between highest disease duration (regardless of other variables) and higher TBS( $p=0.103)$. Lymphocyte count added to 